\title{
Glassy states: the free Ising model on a tree
}

\author{
Daniel Gandolfo, ${ }^{1}$ Christian Maes, ${ }^{2}$ Jean Ruiz, ${ }^{1}$ and Senya Shlosman ${ }^{1,3,4}$ \\ ${ }^{1}$ Aix-Marseille and Université de Toulon, CNRS, CPT, Marseille \\ ${ }^{2}$ Instituut voor Theoretische Fysica, KU Leuven \\ ${ }^{3}$ Skolkovo Institute of Science and Technology \\ ${ }^{4}$ Institute of Information Transmission Problems, RAS, Moscow
}

\begin{abstract}
We consider the ferromagnetic Ising model on the Cayley tree and we investigate the decomposition of the free state into extremal states below the spin glass temperature. We show that this decomposition has uncountably many components. The tail observable showing that the free state is not extremal is related to the Edwards-Anderson parameter, measuring the variance of the (random) magnetization obtained from drawing boundary conditions from the free state.
\end{abstract}

\section{INTRODUCTION}

A principal static feature of the spin glass phase is the presence of infinitely many pure states; see the discussion in [9] and the references therein. In the present paper we identify the same phenomenon in the ferromagnetic Ising model on the tree, i.e., without randomness in the interaction. We show that a very natural state - the free state of the Ising model - which is not a pure state below the spin-glass temperature, has a decomposition into extremal states which involves uncountably many extremal states. That is why we call this state 'glassy'. Our result answers an older question of Arnout Van Enter.

The next section contains the more detailed question with some notations and definitions. Our main decomposition result is given in Section 3. The remaining sections contain further details and proofs.

\section{NOTATIONS AND DEFINITIONS}

Let $\mathcal{T}_{k}=(V, E)$ be the Cayley tree with branching ratio $k \geq 2$. We consider the nearest neighbor Ising model, where spins $\sigma_{x}= \pm 1$ have a Gibbs distribution at temperature $T=$ 
$1 / \beta$ with boundary condition $\eta$ in a finite volume $\Lambda$ given by

$$
\mu(\sigma)=Z^{-1} \exp \left\{\beta \sum_{\langle x, y\rangle} J_{x y} \sigma_{x} \sigma_{y}+\beta \sum_{\langle x, y\rangle} J_{x y} \sigma_{x} \eta_{y}\right\}
$$

Both sums runs over nearest neighbors pairs, the first being over the pairs $x \in \Lambda, y \in \Lambda$, and the second one runs over sites $x \in \Lambda, y \notin \Lambda$. The infinite volume Gibbs distributions are obtained as limit points when $\Lambda$ grows to cover all the vertices of the tree. The set of Gibbs distributions for a fixed temperature and interaction is convex, and its extreme points are called pure states: they cannot be decomposed into other states.

In the ferromagnetic case we put $J_{x y}=1$, and in the spin-glass model the interaction is random $J_{x y}= \pm 1$ with probability $1 / 2$ independently for any pair $\langle x, y\rangle$. We can also consider the random interaction

$$
J_{x y}=\left\{\begin{array}{lll}
-1 & \text { with probability } & p \\
+1 & \text { with probability } & 1-p
\end{array}\right.
$$

which interpolates between the ferromagnetic model $p=0$ and the spin-glass model $p=1 / 2$.

It is known that in the ferromagnetic case the phase transition happens at the critical temperature $T_{\text {cr }}=1 / \operatorname{arctanh}(1 / k)$. For $T>T_{\text {cr }}$ there is a unique infinite volume Gibbs distribution and below that critical temperature the spontaneous magnetization $m^{*}(T)$ becomes nonzero, while plus and minus boundary conditions give rise to different states:

$$
\left\langle\sigma_{0}\right\rangle_{ \pm}^{T}= \pm m^{*}(T), \quad m^{*}(T)>0 \text { iff } T<T_{\mathrm{cr}}
$$

We use the convention that the boundary condition by which the infinite volume Gibbs state is obtained is indicated by a subscript to the expectation $\langle\cdot\rangle$, and superscripts will be used to indicate further parameters like temperature or the choice of model in (2).

There is yet another special temperature, $T_{\mathrm{SG}}=1 / \operatorname{arctanh}(1 / \sqrt{k})$, called the spin-glass temperature. It will often appear in what follows below, and it has various interpretations. For the ferromagnetic model it is known that the free state, the infinite volume Gibbs distribution obtained by putting $\eta \equiv 0$ in (1) is extreme for $T>T_{\mathrm{SG}}$ while it is not for $T<T_{\mathrm{SG}}$; see [1, [3]. Hence the question that motivates the present paper: what is the decomposition of the free state $\langle\cdot\rangle_{\varnothing}^{T}$ (at these lower temperatures) into extremal ones? 
Part of the question is also to understand why that extremality of the free state exactly stops at the spin-glass temperature, which in its origin characterizes a transition to glassy behavior in the spin-glass model. For example consider the spin-glass state obtained via plus-boundary conditions and look at the (random) magnetization:

$$
\left\langle\sigma_{0}\right\rangle_{+}^{T, \mathrm{SG}}=m\left(T,\left\{J_{x y}\right\}\right)
$$

It depends on the independent random variables $J_{x y}$, which take values \pm 1 with equal probability. The spin-glass temperature $T_{S G}$ is that temperature below which $m\left(T,\left\{J_{x y}\right\}\right)$ is a fluctuating quantity with a non-trivial distribution. For example, the second moment, the Edwards-Anderson parameter, is positive there only:

$$
q_{E A}(T)=\mathbb{E}\left[m^{2}\left(T,\left\{J_{x y}\right\}\right)\right]>0 \quad \text { iff } T<T_{\mathrm{SG}},
$$

where the expectation $\mathbb{E}[\cdot]$ is taken over the randomness $\left\{J_{x y}\right\}$.

\section{DECOMPOSITION OF THE FREE STATE}

In this section we present the decomposition of the free state into pure states and we explain that a continuum of them enters into it, at least at low temperatures. Presumably, it is the case for all temperatures in the spin-glass region.

For any Gibbs state $\mu$ corresponding to temperature $T$ we have that

$$
\mu(\cdot)=\int \mu(d \sigma)\left[\langle\cdot\rangle_{\sigma}^{T}\right]
$$

where $\sigma$ is a spin configuration drawn from $\mu$ and used as boundary condition at infinity. The Gibbs distributions $\langle\cdot\rangle_{\sigma}^{T}$ are $\mu$-almost surely extreme. Hence, we have here a decomposition of $\mu$ into extreme Gibbs distributions, but obviously the states $\langle\cdot\rangle_{\sigma}^{T}$ might well be the same for different $\sigma$-s. Nevertheless this decomposition is nontrivial when $\mu$ is not extremal. It remains then to see how many and which different extremal states we get.

For the Ising model free state $\mu_{\varnothing}^{T}$ we will use the Edwards-Sokal representation, [7]:

$$
\mu_{\varnothing}^{T}(\cdot)=\mathbb{E}_{q(T)}\left(\mu^{E S}(\cdot \mid n)\right)
$$

(In contrast to the more standard notation we prefer here to call $q(T)=1-\tanh 1 / T$ the probability of removed (or closed) bonds.) On the tree, the random cluster measure is generated by independent bond percolation and $n$ is the resulting random bond configuration 
over which we take the expectation $\mathbb{E}_{q}$. The open bonds generate a partition of the tree into maximal connected components. The measure $\mu^{E S}(d \sigma \mid n)$ is supported by the spin configurations $\sigma$ which are constant on each connected component as specified by $n$; these constants take values \pm 1 , independently with probability $1 / 2$.

We can rewrite (3) on the tree by ordering the components using the following definitions. Let $D$ be a subset of bonds of the tree $\mathcal{T}_{k}$, and consider the two Ising spin configurations $\sigma^{D, \pm}$ on $\mathcal{T}_{k}$ defined as:

$$
\sigma_{0}^{D,+}=+1, \quad \sigma_{0}^{D,-}=-1
$$

and

$$
\begin{array}{ll}
\sigma_{x}^{D, \pm}=-\sigma_{y}^{D, \pm} & \text { for }(x, y) \in D \\
\sigma_{x}^{D, \pm}=+\sigma_{y}^{D, \pm} & \text { for }(x, y) \notin D
\end{array}
$$

That is, we fix the value of the spin at the root (say 0 ) to be +1 or -1 , and the nearest neighbor spins alternate iff on sites that belong to the set $D$.

By $\langle\cdot\rangle_{\sigma D, w}^{T}$ we denote the Gibbs state of the ferromagnetic Ising model at inverse temperature $T$ with the boundary condition $\sigma^{D, \omega}$ where $\omega= \pm 1$ corresponds to the way the spin at the origin is chosen.

Let $p \in(0,1)$. Take the set $D$ to be random: every bond decides to be in $D$ with probability $p$ independently of the other bonds. Denote by $\mathbb{E}_{p}$ the expectation with respect to that process.

Proposition III.1. The following decomposition of the free state for the ferromagnetic Ising model on the tree holds for all temperatures $T$ :

$$
\langle\cdot\rangle_{\varnothing}^{T}=\frac{1}{2} \mathbb{E}_{p(T)}\left[\langle\cdot\rangle_{\sigma^{D,+}}^{T}+\langle\cdot\rangle_{\sigma^{D,-}}^{T}\right]
$$

where

$$
p(T)=\frac{1}{2}[1-\tanh 1 / T]
$$

Proof. We apply the Edwards-Sokal representation (3) of the Ising model. Start by noting that

$$
\langle\cdot\rangle_{\varnothing}^{T}=\mathbb{E}_{q(T)}\left(\mu^{E S}(\cdot \mid n)\right)=\mathbb{E}_{q(T)} \int\langle\cdot\rangle_{\sigma}^{T} \mu^{E S}(d \sigma \mid n)
$$


here follows some more explanation as requested by the referee: Consider now for a given bond collection $n$ the atomic measure $\mu^{ \pm}(d \sigma \mid n)=\frac{1}{2}\left(\delta_{\sigma^{n+}}+\delta_{\sigma^{n-}}\right)$, which to $n$ assigns two configurations defined by the relations (4), each with probability $\frac{1}{2}$. In other words, fixing the spin of the origin and fixing $n$ determines all the other spin values, where in particular neighboring connected components of open bonds alternate their spin. However the resulting spin configuration would have the same distribution as in the Edwards-Sokal representation with twice as large probability of closed bonds: with $p(T)=q(T) / 2$,

$$
\mathbb{E}_{q(T)}\left[\mu^{E S}(d \sigma \mid n)\right]=\mathbb{E}_{p(T)}\left[\mu^{ \pm}(d \sigma \mid n)\right]
$$

Bringing all that together we conclude that on the tree (3) reduces to the formula (5).

To see that this decomposition (5) is non-trivial for $T<T_{\mathrm{SG}}$ it suffices to show that when $T<T_{\mathrm{SG}},\langle\cdot\rangle_{\sigma^{D,+}}^{T} \neq\langle\cdot\rangle_{\sigma^{D,-}}^{T}$ for $\mathbb{E}_{p(T)^{-}}$-typical sets $D$. That follows from the fact that

$$
\mathbb{E}_{p(T)}\left[\langle\cdot\rangle_{\sigma^{D,+}}^{T}\right]>0>\mathbb{E}_{p(T)}\left[\langle\cdot\rangle_{\sigma D,-}^{T}\right]
$$

The inequality (6) is proven in Section VI by recursion techniques.

Of course, the states $\langle\cdot\rangle_{\sigma^{D,+}}^{T}$ may coincide for different sets $D$ - this is the case at high temperature. However It is known that at low temperatures there is a continuum of different states $\langle\cdot\rangle_{\sigma^{D,+}}^{T}$ as we vary over the sets $D$. That is the result of Theorem 3 in [6]. Indeed, at low temperatures the sets $D$ are composed of rare bonds and thus define stable ground states and mutually singular low-temperature states (with probability one).

Remark: At all temperatures $T$ the free state two-point function $\left\langle\sigma_{0} \sigma_{x}\right\rangle_{\varnothing}^{T} \rightarrow 0$ goes to zero when $x$ goes to infinity. Yet, for $T<T_{\mathrm{SG}}$ the free state is not extreme. In particular, the magnetization in increasingly large volumes has a variance that does not go to zero with the size of the volume. The interesting tail observable which shows that the free state is not extreme is related to the Edwards-Anderson parameter. Here is the simplest version: take the magnetization

$$
M(\tau)=\left\langle\sigma_{0}\right\rangle_{\tau}^{T}
$$

in the infinite volume Gibbs distribution with boundary condition $\tau$; that $\tau$ is drawn from the free state at temperature $T$. For $T<T_{\mathrm{SG}}$ the random variable $M(\tau)$ has a non-trivial distribution. 


\section{DOUBLE TEMPERATURE ISING MODEL}

We already mentioned in the introduction that the phase diagram of the ferromagnetic Ising model is essentially determined by the critical temperature $T_{\mathrm{cr}}=1 / \operatorname{arctanh}(1 / k)$, and the spin glass temperature $T_{\mathrm{SG}}=1 / \operatorname{arctanh}(1 / \sqrt{k}) \cdot \mathbf{A}$ clarification of the situation can however be obtained by enlarging the objects in (5) into a two-temperature setting. We consider the two-temperature states

$$
\nu\left(T_{1}, T_{2}\right):=\langle\cdot\rangle_{\sigma^{D\left(T_{1}\right),+}}^{T_{2}}
$$

which is the infinite volume Gibbs distribution at temperature $T_{2}$ with the boundary condition taken to be the spin configuration (4) where $D$ is drawn from $\mathbb{E}_{p\left(T_{1}\right)}$, the Bernoulli bond percolation process with parameter $1-p\left(T_{1}\right)$. Note that (5) contains these states $\nu(T, T)$ with $T_{1}=T_{2}=T$, and that is why it is useful to speak about the temperature $T_{1}$ but of course the relevant parameter is the density $p\left(T_{1}\right)$. The following is therefore presented in the $(p, T)$-plane, which is also the setting of [5].

Consider the curve

$$
\mathcal{T}_{\mathrm{SG}}(p)=\max \left\{\frac{1}{\operatorname{arctanh}\left[\frac{1}{k(1-2 p)}\right]}, 0\right\} .
$$

Note that $\mathcal{T}_{\mathrm{SG}}(p)>0$ when $k(1-2 p)>1$.

Proposition IV.1. For any positive temperature $T>0$ and parameter $0 \leq p \leq 1 / 2$,

1. When $T \geq \mathcal{T}_{\mathrm{SG}}(p)$, the expected local magnetization of the random Gibbs states $\langle\cdot\rangle_{\sigma^{D, \omega}}^{T}$ vanish,

$$
\mathbb{E}_{p}\left(\left\langle\sigma_{x}\right\rangle_{\sigma^{D, \pm}}^{T}\right)=0
$$

2. When $T<\mathcal{T}_{\mathrm{SG}}(p)$,

$$
\mathbb{E}_{p}\left(\left\langle\sigma_{x}\right\rangle_{\sigma^{D,+}}^{T}\right)=-\mathbb{E}_{p}\left(\left\langle\sigma_{x}\right\rangle_{\sigma^{D,-}}^{T}\right)>0
$$

Let us now look to the second moment, $\mathbb{E}_{p}\left(\left[\left\langle\sigma_{x}\right\rangle_{\sigma^{D, \pm}}^{T}\right]^{2}\right)$, which is called the EdwardsAnderson parameter.

Proposition IV.2. For the random Gibbs state $\langle\cdot\rangle_{\sigma^{D, \pm}}^{T}$ 


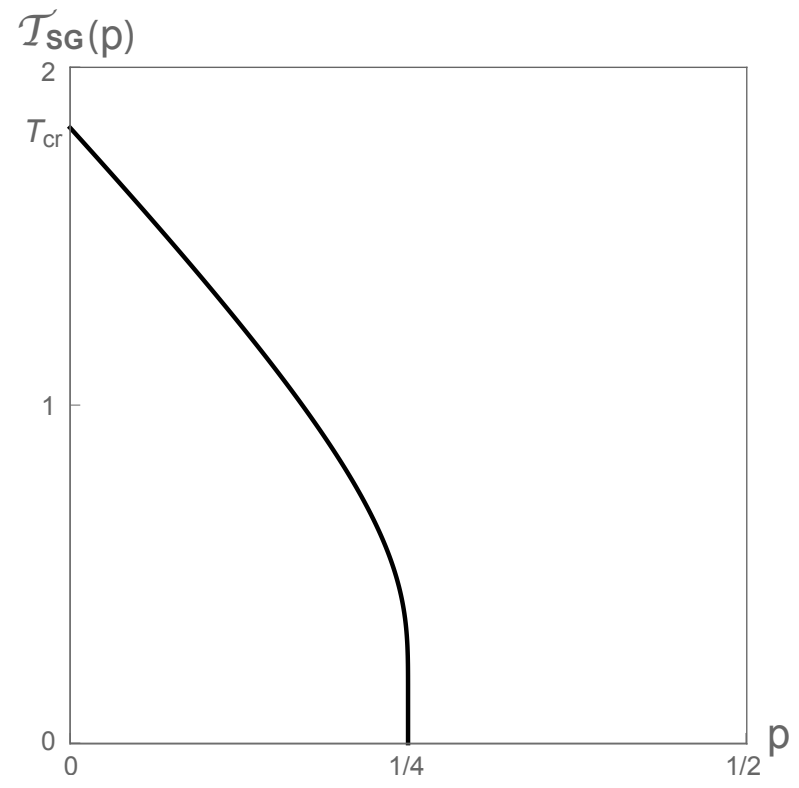

FIG. 1: The curve $\mathcal{T}_{\mathrm{SG}}(p)$.

1. If $T \geq T_{\mathrm{SG}}$ and $T \geq \mathcal{T}_{\mathrm{SG}}(p)$, then

$$
\mathbb{E}_{p}\left(\left[\left\langle\sigma_{x}\right\rangle_{\sigma^{D, \pm}}^{T}\right]^{2}\right)=0
$$

2. Otherwise, for any other temperature $T>0$ :

$$
\mathbb{E}_{p}\left(\left[\left\langle\sigma_{x}\right\rangle_{\sigma^{D, \pm}}^{T}\right]^{2}\right)>0
$$

From Fig. 2 it is clear that the model shows an interesting and non-trivial behavior, even on the line

$$
T_{1}=\infty
$$

It was treated in the paper [2] of Pavel Bleher. More details on the paper by Bleher??? To understand the nature of the spin-glass temperature, we remark that the composition $\mathcal{T}_{\mathrm{SG}}(p(T))$, which we abbreviate as

$$
\mathcal{T}_{\mathrm{SG}}(T):=\mathcal{T}_{\mathrm{SG}}(p(T))=\frac{1}{\operatorname{arctanh}\left(\frac{1}{k \tanh (1 / T)}\right)}
$$

is an involution: $\mathcal{T}_{\mathrm{SG}}\left(\mathcal{T}_{\mathrm{SG}}(T)\right)=T$. In particular,

$$
\mathcal{T}_{\mathrm{SG}}(0)=T_{c r}, \quad \mathcal{T}_{\mathrm{SG}}\left(T_{c r}\right)=0, \quad \mathcal{T}_{\mathrm{SG}}\left(T_{S G}\right)=T_{S G}
$$

Proofs of the propositions are in Section VI. 


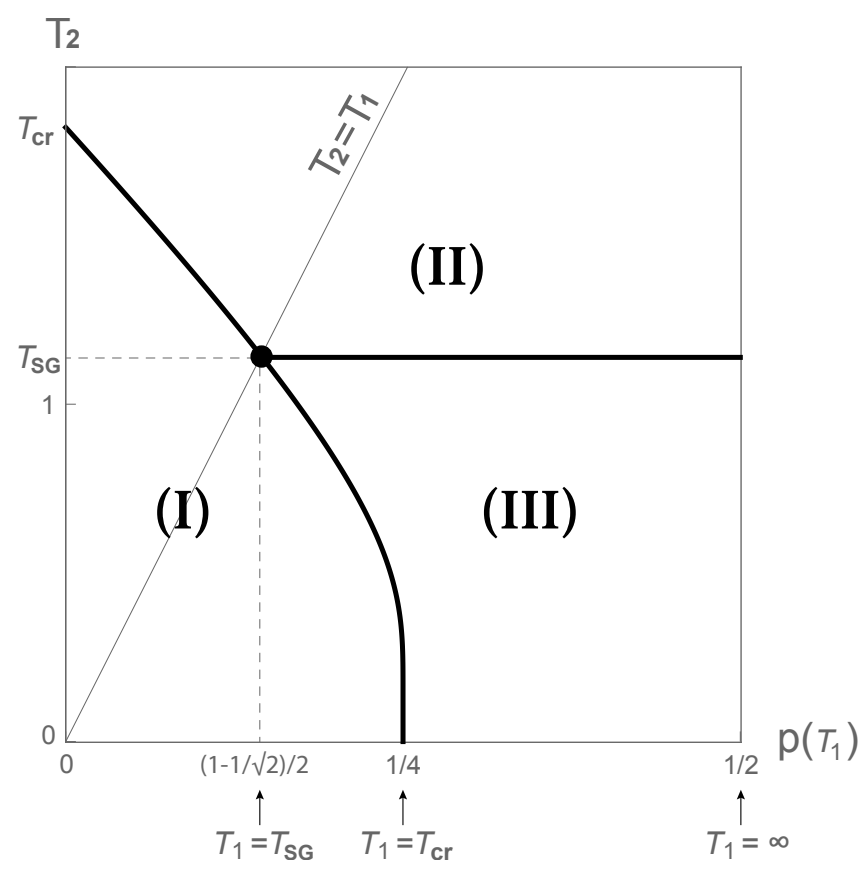

FIG. 2: Phase diagram. Phases I, II, III correspond to the behavior in Proposition IV.1, IV.2.

\section{BETWEEN FERROMAGNETIC AND RANDOM BOND ISING MODELS}

Let us recall the equivalence between the Ising model with random boundary conditions and the spin-glass Ising model; see e.g. [4].

For a fixed vertex $x^{0} \in V$, the root, consider the sphere $W_{n}$ and the ball of radius $n$

$$
\begin{aligned}
& W_{n}=\left\{x \in V: d\left(x, x^{0}\right)=n\right\} \\
& V_{n}=\left\{x \in V: d\left(x, x^{0}\right) \leq n\right\}
\end{aligned}
$$

Remember the definition of the two Ising spin configurations $\sigma^{D, \pm}$ around (4). Denote the corresponding ferromagnetic Gibbs states at temperature $T$ in the volume $V_{n}$ and with the boundary condition $\sigma^{D, \pm}$ by

$$
\mu_{\sigma^{D, \omega}}^{n, T}
$$

with $\omega= \pm 1$.

Consider next the spin-glass Ising model with the following fixed couplings

$$
J_{x y}=\left\{\begin{array}{lll}
-1 & \text { if } & (x, y) \in D \\
+1 & \text { if } & (x, y) \notin D
\end{array}\right.
$$


and denote by

$$
\mu_{\omega}^{J, T, n}
$$

the corresponding Gibbs state at temperature $T$ in the volume $V_{n}$ with the boundary conditions \pm 1 (that is either the + boundary condition or the - boundary conditions). Note however that the states $\mu_{\sigma^{D,+}}^{n, T}$ and $\mu_{+}^{J, T, n}$ are the same states up to a gauge transformation. The same is true for the states $\mu_{\sigma^{D,-}}^{n, T}$ and $\mu_{-}^{J, T, n}$.

Indeed, observe that in both cases with $D,+$ or $D,-$,

$$
\sigma_{x}^{D}=(-1)^{\mathrm{fr}_{D}(0 \rightarrow x)} \sigma_{0}^{D}
$$

where the quantity $\operatorname{fr}_{D}(0 \rightarrow x)$ is the number of frustrated bonds over which the spins alternate of the configuration $D$ in the path starting from the root 0 and ending to the vertex $x$. The Gibbs state $\mu_{\sigma^{D}}^{n, T}$ is given by

$$
\mu_{\sigma^{D}}^{n, T}(\eta)=\left(1 / Z_{n}\right) \exp \left\{\beta \sum_{\langle x, y\rangle \subset V_{n}} \eta_{x} \eta_{y}+\beta \sum_{x \in W_{n}} \sum_{y \in S(x)} \eta_{x} \sigma_{y}^{D}\right\}
$$

where $S(x)$ is the collection of neighbors of $\mathbf{x}$ outside the volume $V_{n}$. But since

$$
\sigma_{y}^{D}=\prod_{\langle z, t\rangle: 0 \rightarrow y} J_{z t} \sigma_{0}^{D}
$$

the right-hand side of (11) will read

$$
\left(1 / Z_{n}\right) \prod_{\langle x, y\rangle \subset V_{n}} e^{\beta J_{x y} \eta_{x} \eta_{y}} \prod_{x \in W_{n}} \prod_{y \in S(x)} e^{\beta \eta_{x} J_{x y} \sigma_{0}^{D}}
$$

which we recognize as the Gibbs states of the spin-glass model with fixed couplings (9) with + or - boundary condition (depending to the way we choose the spin $\sigma_{0}^{D}$ of the root).

Conversely, let us start with the spin glass model defined by the Gibbs distribution (10). Then consider the gauge transformation

$$
\begin{gathered}
\sigma_{x} \rightarrow \sigma_{x}^{\prime}=\sigma_{x} s_{x} \\
J_{x y} \rightarrow J_{x y}^{\prime}=J_{x y} s_{x} s_{y}
\end{gathered}
$$

and choose

$$
s_{x}=\sigma_{x}^{D}
$$


Observe that

$$
\sum_{\langle x, y\rangle} J_{x y}^{\prime} \sigma_{x}^{\prime} \sigma_{y}^{\prime}=\sum_{\langle x, y\rangle} J_{x y} \sigma_{x} \sigma_{y}
$$

As can be seen from 12 , this gauge transformation maps the Gibbs distribution $\mu_{\omega}^{J, n, T}$ into the Gibbs distribution $\mu_{\sigma^{D, \omega}}^{n, T}$.

Next, let the set of bonds $D$ be random. That is for $p \in(0,1)$, let $D(p)$ be the random configuration where bonds are marked independently. Denote by $n_{p}$ this process. The ferromagnetic Gibbs state (8) then becomes a ferromagnetic Ising model with random boundary conditions

$$
\mu_{\sigma^{D(p), \pm}}^{n, T}
$$

As follows from the above, that state is equivalent to the state of the spin-glass model with fixed boundary condition $\omega= \pm 1$ and random couplings $J_{x y}$ given by (2), or equivalently by

$$
J_{x y}=\left\{\begin{array}{lll}
-1 & \text { if } & (x, y) \in D(p) \\
+1 & \text { if } & (x, y) \notin D(p)
\end{array}\right.
$$

We denote this state by

$$
\mu_{\omega}^{n, T, J(p)}
$$

Obviously, for the expectations with respect to the process $n_{p}$ we have

$$
\mathbb{E}_{p}\left(\mu_{\sigma^{D(p), \pm}}^{n, T}\right)=\mathbb{E}_{p}\left(\mu_{\omega}^{n, T, J(p)}\right)
$$

Therefore various problems of the ferromagnetic Ising model with random boundary conditions can be translated in terms of the spin-glass model provided one proceeds as follows. Consider a very large box of size $n$ and compute quantities such as the local magnetization for the sites far away from the boundary, i.e. inside a box of size $r$. Then, take first the limit when $n \rightarrow \infty$ and after that take the limit $r \rightarrow \infty$. However contrary to what was done in [4] where computations are formulated in terms of random boundary conditions to get results for the spin-glass model, here we prefer to adopt the opposite direction. The reason for doing so is that the latter formulation allows to immediately catch that the recursive equations of the Proposition V.1 below reduces, in case e.g. of the singlesite magnetization, to a one-dimensional recursion. Obviously both formulations lead to the same conclusions. 
Observe then, that in term of the boundary fields

$$
h_{x}=\beta \sum_{y \in S(x)} J_{x y} \omega
$$

the Gibbs distributions $\mu_{\omega}^{n, T, J(p)}$ in 13 read

$$
\mu_{\omega}^{n, T, J(p)}\left(\sigma_{n}\right)=Z_{n}^{-1} \exp \left\{\beta \sum_{\langle x, y\rangle \subset V_{n}} J_{x y} \sigma_{x} \sigma_{y}+\sum_{x \in W_{n}} h_{x} \sigma_{x}\right\}
$$

for the spin configurations $\sigma_{n} \in\{+1,-1\}^{V_{n}}$ of the ball $V_{n}$.

Introduce next, for real valued boundary fields $h_{x}, x \in V$, the Gibbs distributions

$$
\mu_{n}\left(\sigma_{n}\right)=Z_{n}^{-1} \exp \left\{\beta \sum_{\langle x, y\rangle \subset V_{n}} J_{x y} \sigma_{x} \sigma_{y}+\sum_{x \in W_{n}} h_{x} \sigma_{x}\right\}
$$

Those probability distributions are said compatible if when summing over the spins $\sigma_{x}=$ $\{+1,-1\}$ of the sphere $W_{n}$ we have

$$
\sum_{\sigma_{x}= \pm 1, x \in W_{n}} \mu_{n}\left(\sigma_{n}\right)=\mu_{n-1}\left(\sigma_{n-1}\right)
$$

Proposition V.1. (P. Bleher [1]). The probability distributions $\mu_{n}$ are compatible if and only if for any $x$ the following recursive equations hold

$$
h_{x}=\sum_{y \in S(x)} f\left(h_{y}\right)
$$

where

$$
f(h)=\operatorname{arctanh}[\theta \tanh (h)]
$$

and

$$
\theta=\tanh (1 / T)
$$




\section{RECURSION METHOD}

\section{A. The magnetization}

The recursive equations (16) for the boundary fields defined by 14 read also in case $\omega=+1$ :

$$
\begin{aligned}
\left\langle\sigma_{x}\right\rangle & =\tanh \left\{\sum_{y \in S(x)} \operatorname{arctanh}\left(\theta J_{x y}\left\langle\sigma_{y}\right\rangle\right)\right\} \\
& =\tanh \left\{\sum_{y \in S(x)} J_{x y} \operatorname{arctanh}\left(\theta\left\langle\sigma_{y}\right\rangle\right)\right\}
\end{aligned}
$$

where we used the notation

$$
\left\langle\sigma_{x}\right\rangle=\tanh h_{x}
$$

Notice that $\left\langle\sigma_{y}\right\rangle$ and $\left\langle\sigma_{z}\right\rangle$ are the magnetization at sites $y$ and $z$ that they would have if they were disconnected from the site $x$ (the same with $x$ when this site is disconnected from his parent). Notice also that the equality (17) holds true in case $\omega=-1$.

Next, consider the spheres defined in (7), $W_{m}, m=0,1, \ldots, n+1$, entering in the recursion (17).

The following lemma states that the expectations with respect to the random variables $J_{x y}$ of those half-tree magnetization depends only on the level $m$. It provides, by denoting by $E_{m}$ these expectations, a one-dimensional set of recursive equations for these quantities.

Lemma VI.1. Assume that the temperature $T>0$. Then, for the probability distributions $\mu_{\omega}^{n, T, J(p)}$, the expectations $\mathbb{E}_{p}\left(\left\langle\sigma_{x}\right\rangle\right)$ are the same for all the vertices $x$ belonging to the sphere $W_{m}$. Those expectations $E_{m}$ satisfy, in case of degree $k=2$ and for $m=1, \ldots, n$, the recursive equations

$$
E_{m}=F\left(E_{m+1}\right)
$$

with

$$
F(E)=\frac{k(1-2 p) \theta E}{1+\theta^{2} E^{2}}
$$

and $E_{n+1}=+1$. For the probability distributions $\mu_{-}^{n, T, J(p)}$ the same results holds true with $E_{n+1}=-1$. 
Proof. In case of degree $k=2$, the formula (17) gives:

$$
\left\langle\sigma_{x}\right\rangle=\frac{\theta J_{x y}\left\langle\sigma_{y}\right\rangle+\theta J_{x z}\left\langle\sigma_{z}\right\rangle}{1+\theta^{2} J_{x y}\left\langle\sigma_{y}\right\rangle J_{x z}\left\langle\sigma_{z}\right\rangle}
$$

where $y$ and $z$ are the successors of the site $x$. Observe that at the beginning level of recursion $m=n+1$ the quantities $\left\langle\sigma_{x}\right\rangle$ are merely the boundary conditions. Take the boundary condition $\omega=+1$ in which case $\left\langle\sigma_{x}\right\rangle=+1$ for all $x \in W_{n+1}$.

Then, in the first step of recursion, that is for the boundary sites $x \in W_{n}$, the formula (21) reads:

$$
\left\langle\sigma_{x}\right\rangle=\frac{\theta J_{x y}+\theta J_{x z}}{1+\theta^{2} J_{x y} J_{x z}}
$$

Next, let us perform the expectation $\mathbb{E}\left(\left\langle\sigma_{x}\right\rangle\right)$ of this quantity over the random variables $J_{x y}$ and $J_{x z}$. Remembering that those random variables are independent, take value 1 with probability $1-p$ and take value -1 with probability $p$, we get that these expectations at the level $n$ are given by

$$
\begin{aligned}
E_{n} & =\mathbb{P}\left(J_{x y}=1\right) \mathbb{P}\left(J_{x z}=1\right) \frac{2 \theta}{1+\theta^{2}}-\mathbb{P}\left(J_{x y}=-1\right) \mathbb{P}\left(J_{x z}=-1\right) \frac{2 \theta}{1+\theta^{2}} \\
& +\mathbb{P}\left(J_{x y}=1\right) \mathbb{P}\left(J_{x z}=-1\right) \frac{\theta-\theta}{1-\theta^{2}}-\mathbb{P}\left(J_{x y}=-1\right) \mathbb{P}\left(J_{x z}=1\right) \frac{\theta-\theta}{1-\theta^{2}} \\
& =(1-p)^{2} \frac{2 \theta}{1+\theta^{2}}-p^{2} \frac{2 \theta}{1+\theta^{2}} \\
& =\frac{2 \theta(1-2 p)}{1+\theta^{2}}
\end{aligned}
$$

Note that we used here that $\theta^{2}<1$, which excludes the zero temperature case; that can be treated separately however, as is done in [2].

Furthermore, it is obvious that $\mathbb{E}\left(\left\langle\sigma_{x}\right\rangle\right)$ are the same for all the sites $x$ belonging to the sphere $W_{n}$. Notice also that all moments $\mathbb{E}\left(\left\langle\sigma_{x}\right\rangle^{p}\right)$ at that level $n$ are the same.

Let us next consider the second step of the recursion. In this step for a site $x \in W_{n-1}$ we know that the expectation of the magnetization of its successors are given by the above formula. Therefore for the sites $x \in W_{n_{-1}}$ the recursion (21) reads:

$$
\left\langle\sigma_{x}\right\rangle=\frac{\left(J_{x y}+J_{x z}\right) \theta E_{n}}{1+J_{x y} J_{x z} \theta^{2} E_{n}^{2}}
$$


By taking in the above equation the expectation over the random variables $J_{x y}$ and $J_{x z}$ and using again that $J_{x y}$ and $J_{x z}$ are independent, we get

$$
\begin{aligned}
E_{n-1} & =\mathbb{P}\left(J_{x y}=1\right) \mathbb{P}\left(J_{x z}=1\right) \frac{2 \theta E_{n}}{1+\theta^{2} E_{n}^{2}}-\mathbb{P}\left(J_{x y}=-1\right) \mathbb{P}\left(J_{x z}=-1\right) \frac{2 \theta E_{n}}{1+\theta^{2} E_{n}^{2}} \\
& +\mathbb{P}\left(J_{x y}=1\right) \mathbb{P}\left(J_{x z}=-1\right) \frac{\left(\theta E_{n}-\theta E_{n}\right)}{1-\theta^{2} E_{n}^{2}} \\
& -\mathbb{P}\left(J_{x y}=-1\right) \mathbb{P}\left(J_{x z}=1\right) \frac{\left(\theta E_{n}-\theta E_{n}\right)}{1-\theta^{2} E_{n}^{2}} \\
& =(1-p)^{2} \frac{2 \theta E_{n}}{1+\theta^{2} E_{n}^{2}}-p^{2} \frac{2 \theta E_{n}}{1+\theta^{2} E_{n}^{2}} \\
& =\frac{2 \theta(1-2 p) E_{n}}{1+\theta^{2} E_{n}^{2}}
\end{aligned}
$$

For the next steps of recursion we proceed analogously to obtain the recursion formula (19).

Let us then turn to the

\section{Proof of Proposition IV.1}

The function $F$ in Lemma VI.1 giving the recursion of expectations of our process has the following properties. It is an odd and increasing function, concave for positive $E$, and convex for negative $E$. In addition, it has the slope

$$
k \theta(1-2 p)
$$

at the origin, and takes finite values at $E= \pm 1$.

When $k \theta(1-2 p)<1, E=0$ is the unique solution of the equation $E=F(E)$. Under this condition, the recursion leads to that solution so that the half-tree magnetization vanishes.

Whenever $k \theta(1-2 p)>1$, the equation $E=F(E)$ has two stable fixed points and the unstable solution 0 . Under this condition, the recursion leads obviously to the positive solution if we start with the boundary condition $\omega=+1$ and to the negative one if we take $\omega=-1$.

As a consequence we get that in the limit $n \rightarrow \infty$ the expectations of half-tree magnetization at the root goes to the positive or negative solution according to the choice of the boundary conditions to be +1 or -1 . Fig. 3 shows a plot of the solution $\mathcal{E}^{\star}$ of the fixed point equation $E=F(E)$. 


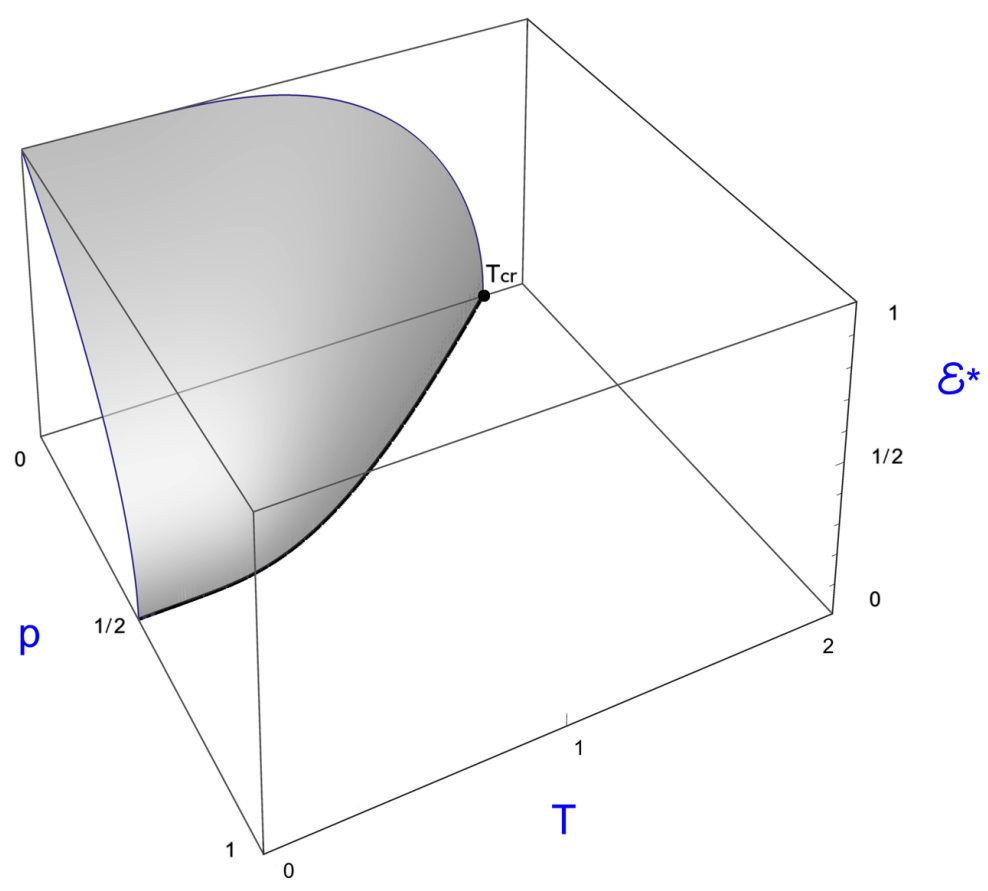

FIG. 3: The first moment.

To compute these expectations on the full tree is easy. Look at the neighbors of the root, say $y, z, t$. We have by recursion

$$
\begin{aligned}
\left\langle\sigma_{0}\right\rangle & =\tanh \left\{J _ { 0 y } \operatorname { a r c t a n h } \left(\theta\left\langle\sigma_{y}\right\rangle+J_{0 z} \operatorname{arctanh}\left(\theta\left\langle\sigma_{z}\right\rangle+J_{0 t} \operatorname{arctanh}\left(\theta\left\langle\sigma_{t}\right\rangle\right\}\right.\right.\right. \\
& =\frac{\left(J_{0 y}+J_{0 z}+J_{0 t}\right) \theta E_{1}+J_{0 x} J_{0 z} J_{0 t} \theta^{3} E_{1}^{3}}{1+\left(J_{0 y} J_{0 z}+J_{0 z} J_{0 t}+J_{0 t} J_{0 v}\right) \theta^{2} E_{1}^{2}}
\end{aligned}
$$

where $\left\langle\sigma_{y}\right\rangle=\left\langle\sigma_{y}\right\rangle=\left\langle\sigma_{y}\right\rangle=E_{1}$ are the expectations of the half tree magnetization at the level 1. Here we used the equation

$$
\tanh (a+b+c)=\frac{\tanh a+\tanh b+\tanh c+\tanh a \tanh b \tanh c}{1+\tanh a \tanh b+\tanh b \tanh c+\tanh c \tanh a}
$$

It is obvious that expectation $\mathbb{E}\left(\left\langle\sigma_{0}\right\rangle\right)$ will behave in the limit $n \rightarrow \infty$ as the expectations of the half tree magnetization.

It is easy to understand that this holds also true for any site provided we proceed as described above. Namely consider a very large box of size $n$ and compute the local magnetization for the sites far away from the boundary, i.e. inside a box $r$. Then, take first the limit when $n \rightarrow \infty$ and after that take the limit $r \rightarrow \infty$. 


\section{B. The second moment: Edwards-Anderson parameter.}

Consider again the spheres defined in (7), $W_{m}, m=0,1, \ldots, n+1$, entering in the recursion 17 .

The following lemma says that the expectations with respect to the random variables $J_{x y}$ of the square of half-tree magnetization depends only on the level $m$. It provides, denoting by $D_{m}$ these expectations, a one-dimensional recursive equation for these quantities.

Lemma VI.2. Assume that the temperature $T=1 / \beta$ is positive, then for the probability distributions $\mu_{\omega}^{n, T, J(p)}$, the expectations $\mathbb{E}_{p}\left(\left\langle\sigma_{x}\right\rangle^{2}\right)$ are the same for all the vertices $x$ belonging to the level $m$. These expectations $D_{m}$ satisfy, in case of degree $k=2$ and for $m=1, \ldots, n$, the recursive equations

$$
D_{m}=G\left(D_{m+1}, E_{m+1}\right)
$$

where

$$
G(D, E)=[1-2 p(1-p)] \frac{2 \theta^{2}\left(D+E^{2}\right)}{1+2 \theta^{2} E_{m+1}^{2}+\theta^{4} D^{2}}+2 p(1-p) \frac{2 \theta^{2}\left(D-E^{2}\right)}{1-2 \theta^{2} E^{2}+\theta^{4} D^{2}}
$$

and the $E_{m}$ are given by the recursive equations (19) of Lemma VI.1.

Proof. Recall that at the level $m=n+1$ the quantities $\left\langle\sigma_{x}\right\rangle$ are the boundary conditions so that

$$
\left\langle\sigma_{x}\right\rangle=\left\langle\sigma_{x}\right\rangle^{2}=1
$$

for all sites belonging to the sphere $W_{n+1}$.

In the first step of recursion, that is for the boundary sites $x \in W_{n}$, we know by (21) that at this level:

$$
\left\langle\sigma_{x}\right\rangle=\frac{\theta J_{x y}+\theta J_{x z}}{1+\theta^{2} J_{x y} J_{x z}}
$$

in case $\omega=+1$. Therefore

$$
\left\langle\sigma_{x}\right\rangle^{2}=\frac{2 \theta^{2}\left(1+J_{x y} J_{x z}\right)}{1+2 \theta^{2} J_{x y} J_{x z}+\theta^{4}}
$$

By taking the expectation over the random variables $J_{x y}$ and $J_{x z}$ we get: 


$$
\begin{aligned}
D_{n} & =\mathbb{P}\left(J_{x y}=1\right) \mathbb{P}\left(J_{x z}=1\right) \frac{4 \theta^{2}}{\left(1+\theta^{2}\right)^{2}}-\mathbb{P}\left(J_{x y}=-1\right) \mathbb{P}\left(J_{x z}=-1\right) \frac{4 \theta^{2}}{\left(1+\theta^{2}\right)^{2}} \\
& +\mathbb{P}\left(J_{x y}=1\right) \mathbb{P}\left(J_{x z}=-1\right) \frac{2 \theta^{2}-2 \theta^{2}}{\left(1-\theta^{2}\right)^{2}}-\mathbb{P}\left(J_{x y}=1\right) \mathbb{P}\left(J_{x z}=-1\right) \frac{2 \theta^{2}-2 \theta^{2}}{\left(1-\theta^{2}\right)^{2}} \\
& =(1-p)^{2} \frac{4 \theta^{2}}{\left(1+\theta^{2}\right)^{2}}-p^{2} \frac{4 \theta^{2}}{\left(1+\theta^{2}\right)^{2}} \\
& =(1-2 p) \frac{2 \theta^{2}}{\left(1+\theta^{2}\right)^{2}}
\end{aligned}
$$

Here we used (as in the proof of Lemma VI.1) that the temperature is positive $\left(\theta^{2}<1\right)$.

Let us next consider the second step of the recursion. From the formula (21), giving the recursion of half tree magnetization, we have:

$$
\left\langle\sigma_{x}\right\rangle^{2}=\theta^{2} \frac{\left\langle\sigma_{y}\right\rangle^{2}+\left\langle\sigma_{z}\right\rangle^{2}+2 J_{x y} J_{x z}\left\langle\sigma_{y}\right\rangle\left\langle\sigma_{z}\right\rangle}{1+2 \theta^{2} J_{x y} J_{x z}\left\langle\sigma_{y}\right\rangle\left\langle\sigma_{z}\right\rangle+\theta^{4}\left\langle\sigma_{y}\right\rangle^{2}\left\langle\sigma_{z}\right\rangle^{2}}
$$

But we know in this second step that for the successors $y$ and $z$ of the site $x$ :

$$
\left\langle\sigma_{y}\right\rangle^{2}=\left\langle\sigma_{z}\right\rangle^{2}=D_{n}
$$

and

$$
\left\langle\sigma_{y}\right\rangle=\left\langle\sigma_{y}\right\rangle=E_{n}
$$

Therefore, the above formula (32) reads:

$$
\left\langle\sigma_{x}\right\rangle^{2}=\theta^{2} \frac{2 D_{n}+2 J_{x y} J_{x z} E_{n}^{2}}{1+2 \theta^{2} J_{x y} J_{x z} E_{n}^{2}+\theta^{4} D_{n}^{2}}
$$

Let us then compute for the sites $x \in W_{n-1}$ the expectations $\mathbb{E}\left(\left\langle\sigma_{x}\right\rangle^{2}\right)$ over the random variables $J_{x y}$ and $J_{x z}$ :

$$
\begin{aligned}
D_{n-1} & =\mathbb{P}\left(J_{x y}=+1\right) \mathbb{P}\left(J_{x z}=+1\right) \frac{2 \theta^{2}\left(D_{n}+E_{n}^{2}\right)}{1+2 \theta^{2} E_{n}^{2}+\theta^{4} D_{n}^{2}} \\
& +\mathbb{P}\left(J_{x y}=-1\right) \mathbb{P}\left(J_{x z}=-1\right) \frac{2 \theta^{2}\left(D_{n}+E_{n}^{2}\right)}{1+2 \theta^{2} E_{n}^{2}+\theta^{4} D_{n}^{2}} \\
& +\mathbb{P}\left(J_{x y}=+1\right) \mathbb{P}\left(J_{x z}=-1\right) \frac{2 \theta^{2}\left(D_{n}-E_{n}^{2}\right)}{1-2 \theta^{2} E_{n}^{2}+\theta^{4} D_{n}^{2}} \\
& +\mathbb{P}\left(J_{x y}=-1\right) \mathbb{P}\left(J_{x z}=+1\right) \frac{2 \theta^{2}\left(D_{n}-E_{n}^{2}\right)}{1-2 \theta^{2} E_{n}^{2}+\theta^{4} D_{n}^{2}} \\
& =\left[(1-p)^{2}+p^{2}\right] \frac{2 \theta^{2}\left(D_{n}+E_{n}^{2}\right)}{1+2 \theta^{2} E_{n}^{2}+\theta^{4} D_{n}^{2}}+2 p(1-p) \frac{2 \theta^{2}\left(D_{n}-E_{n}^{2}\right)}{1-2 \theta^{2} E_{n}^{2}+\theta^{4} D_{n}^{2}} \\
& =[1-2 p(1-p)] \frac{2 \theta^{2}\left(D_{n}+E_{n}^{2}\right)}{1+2 \theta^{2} E_{n}^{2}+\theta^{4} D_{n}^{2}}+2 p(1-p) \frac{2 \theta^{2}\left(D_{n}-E_{n}^{2}\right)}{1-2 \theta^{2} E_{n}^{2}+\theta^{4} D_{n}^{2}}
\end{aligned}
$$


The next steps of recursion proceed analogously. The above arguments immediately apply in case $\omega=-1$

\section{Proof of Proposition IV.2}

1. From Proposition IV.1, we know that when $T \geq \mathcal{T}_{\mathrm{SG}}(p)$, the first moment vanishes. Therefore the recursion equation for the second moment will reduce to $\mathbf{i}$ changed the 2 into a $\mathrm{k}$

$$
G(D, E)=G(D, 0)=\frac{k \theta^{2} D}{1+\theta^{4} D_{n}^{2}}
$$

The function $G(D, 0)$ has the following properties. It is increasing and concave, has the slope $k \theta^{2}$ at the origin, and takes finite values at $D=1$.

When $k \theta^{2}<1, D=0$ is the unique solution of the equation $D=G(D, 0)$. Under this condition, the recursion leads to that solution.

Whenever $k \theta^{2}>1$, the equation $D=G(D, 0)$ has a unique positive stable fixed point and the unstable solution 0 . Under this condition, the recursion leads obviously to the positive solution.

The solution $\mathcal{D}^{\star}$ of the fixed-point equation $D=G(D, 0)$ is given by

$$
\mathcal{D}^{\star}=\frac{\sqrt{2 \theta^{2}-1}}{\theta^{2}}
$$

See Fig. 4 for a graphical representation of the solution (when $T \geq \mathcal{T}_{\mathrm{SG}}(p)$ ) and note that the bold curve in the plane $(p, T)$ of Fig. 4 is the involution curve $T=\mathcal{T}_{\mathrm{SG}}(p)$.

2. When $T \leq \mathcal{T}_{\mathrm{SG}}(p)$, the fixed-point equation 19 is readily solved

$$
\mathcal{E}^{\star}=\frac{\sqrt{\theta(2-4 p)-1}}{\theta}
$$

leading to the following expression for $G\left(D, \mathcal{E}^{\star}\right)$

$$
\frac{2(2(p-1) p+1)(1-\theta(D \theta-4 p+2))}{-D^{2} \theta^{4}+\theta(8 p-4)+1}-\frac{4(p-1) p(\theta(D \theta+4 p-2)+1)}{D^{2} \theta^{4}+\theta(8 p-4)+3} .
$$

Equation $D=G\left(D, \mathcal{E}^{\star}\right)$ is a quintic polynomial in $\mathcal{D}^{\star}$ whose solutions can be expressed in terms of special functions, see e.g. [8]. These expressions are too cumbersome to be included here. For the solution of the fixed point equation we have proceeded numerically: see Fig. 4 for a picture of the solution when $T \leq \mathcal{T}_{\mathrm{SG}}(p)$. 
The complete expression for $\mathcal{D}^{\star}$ is given by the union of the solutions for $T \leq \mathcal{T}_{\mathrm{SG}}(p)$ and $T \geq \mathcal{T}_{\mathrm{SG}}(p)$ along a separatrix (see Fig. 4). Note that the projection of this separatrix on the plane $(p, T)$ coincides with the involution curve $\mathcal{T}_{\mathrm{SG}}(p)$.

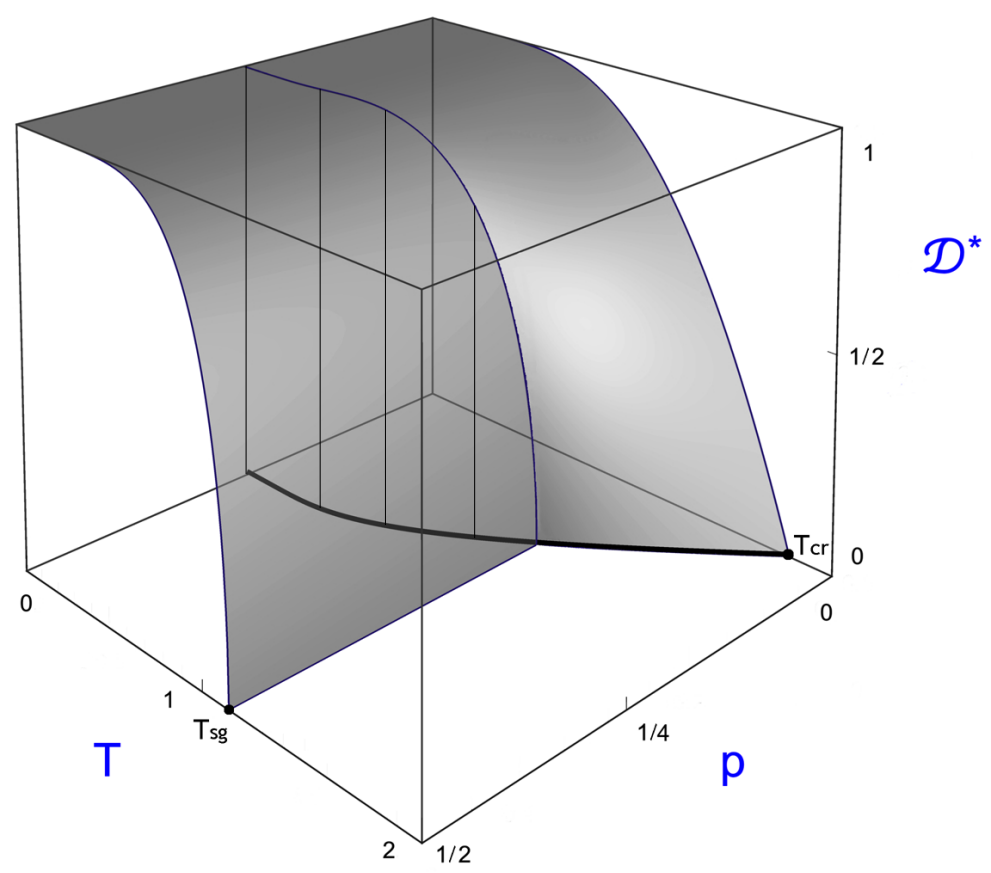

FIG. 4: The second moment.

\section{GENERAL DEGREE REMARKS}

So far we treated the case $k=2$. For the general case and concerning the expectation of magnetization, we can use the formula

$$
\tanh \left(a_{1}+\cdots+a_{k}\right)=\frac{\sum_{\text {odd } p} \sum_{j_{1<j_{2}<\cdots<j_{p}}} \tanh a_{j_{1}} \cdots \tanh a_{j_{p}}}{\sum_{\text {even } p} \sum_{j_{1}<j_{2}<\cdots<j_{p}} \tanh a_{j_{1}} \cdots \tanh a_{j_{p}}}
$$

Using this formula and proceeding as in Lemma VI.1, we get that the recursion formula (23) reads now:

$$
\begin{aligned}
\left\langle\sigma_{x}\right\rangle & =(1 / D)\left(J_{x y_{1}}+J_{x y_{2}}+\cdots+J_{x y_{k}}\right) \theta E_{n} \\
& +(1 / D) \sum_{j_{1}<j_{2}<j_{3}} J_{x y_{j_{1}}} J_{x y_{j_{2}}} J_{x y_{j_{3}}} \theta^{3} E_{n}^{3} \\
& +(1 / D) \sum_{5 \leq p \leq k \text { :odd }} \sum_{j_{1}<j_{2}<\cdots<j_{p}} \prod_{i=1}^{p} J_{x y_{j_{i}}} \theta^{p} E_{n}^{p}
\end{aligned}
$$


with

$$
\begin{aligned}
D & =1 \\
& +\sum_{j_{1}<j_{2}} J_{x y_{j_{1}}} J_{x y_{j_{2}}} \theta^{2} E_{n}^{2} \\
& +\sum_{4 \leq p \leq k \text { :even }} \sum_{j_{1}<j_{2}<\cdots<j_{p}} \prod_{i=1}^{p} J_{x y_{j_{p}}} \theta^{p} E_{n}^{p}
\end{aligned}
$$

Here, $y_{1}, \ldots, y_{k}$ denote the successors of the vertex $x$.

We can next perform the expectations over the random variables $J_{x y_{1}}, \ldots, J_{x y_{k}}$ to get that the function $F(E)$ has the following properties:

1. $F(E)$ is an odd function.

2. $F(E)$ is concave for positive $E$ and convex for negative $E$.

Indeed, there is no need to write down a formula for $F(E)$ to catch the two above properties. However, in this straightforward computation, we still have to assume the condition $\theta^{2}<1$ which excludes the zero temperature case.

Next, we want to know the slope of that function at the origin. Here, it is easy to realize that this slope will be given by the linear term

$$
\left(J_{x y_{1}}+J_{x y_{2}}+\cdots+J_{x y_{k}}\right) \theta E_{n}
$$

Remind that the random variables are independent and that their expectations are given by $1-2 p$. Hence, the slope at the origin of the function $F(E)$ is $k(1-2 p) \theta$.

As a consequence we extend in this way our conclusions to the case of general degree (with analogous arguments for the second moment).

Acknowledgments Part of this work has been carried out in the framework of the Labex Archimede (ANR-11-LABX-0033) and of the A*MIDEX project (ANR-11-IDEX-0001-02), funded by the "Investissements d'Avenir" French Government programme managed by the French National Research Agency (ANR). Part of this work has been carried out at IITP RAS. The support of Russian Foundation for Sciences (project No. 14-50-00150) is gratefully acknowledged. This work was partially supported by the CNRS PICS grant "Interfaces 
aléatoires discrètes et dynamiques de Glauber." CM thanks the hospitality of the CPTLuminy at Marseille.

[1] P.M. Bleher, Extremity of the Disordered Phase in the Ising Model on the Bethe Lattice. Commun. Math. Phys. 128: 411-419 (1990).

[2] P.M. Bleher, The Bethe Lattice spin glass at zero temperature. Ann. Inst. Henri Poicaré. 54: 89-113 (1991).

[3] P.M. Bleher, J. Ruiz et V. Zagrebnov, On the purity of the limiting Gibbs state for the Ising model on the Bethe lattice, J. Stat. Phys. 79, 473-482 (1995).

[4] J.T. Chayes, L. Chayes, J. P. Sethna and D. J. Thouless, A mean field Spin glass with short rang interaction. Commun. Math. Phys. 106: 41-89 (1986).

[5] J.M. Carlson, J.T. Chayes, L. Chayes, J. P. Sethna and D. J. Thouless, Critical behavior of the Bethe lattice spin glass. Euophys. lett. 5: 355-360 (1988).

[6] D. Gandolfo, J. Ruiz, and S.B. Shlosman, A Manifold of Pure Gibbs States of the Ising Model on a Cayley tree. J. Stat. Phys 148, 999-1005 (2012).

[7] H.-O. Georgii, O. Häggström and C. Maes, The random geometry of equilibrium phases, Phase Transitions and Critical Phenomena, vol 18, Eds C. Domb and J.L. Lebowitz (Academic Press, London) pp 1-142, 2001.

[8] R. Bruce King, Beyond the Quartic Equation. Birkhaüser Boston, (1966).

[9] Newman, Charles M., and Daniel L. Stein. Short-range spin glasses: results and speculations. Lecture notes in Mathematics, Springer-Verlag 1900, (2007). 